Classification

Physics Abstracts

83.10D

\title{
Echauffements et pertes rotoriques d'un moteur synchrone autopiloté
}

\author{
D. Milimonfared, M. Parchemin, J. F. Rialland et R. Bonnefille \\ Laboratoire d'Electricité Industrielle, CNAM, 292, rue Saint-Martin, 75141 Paris Cedex 03, France
}

(Reçu le 20 mars 1986, révisé le 30 mai, accepté le 24 juin 1986)

\begin{abstract}
Résumé. - La commande des machines par des convertisseurs statiques entraîne une augmentation des pertes électromagnétiques. Dans cet article, les échauffements rotoriques d'un moteur synchrone autopiloté sont relevés au moyen de thermocouples. Les pertes sont déduites des courbes d'échauffements à l'aide d'un modèle thermique simplifié. La machine testée, d'une puissance apparente nominale de $10 \mathrm{kVA}$ à $3000 \mathrm{tr} \cdot \min ^{-1}$, est à rotor massif et pôles saillants $(2 p=4)$. Celle-ci est alimentée par un commutateur de courants, piloté par la machine dès que la vitesse de rotation est supérieure à $200 \mathrm{tr} . \mathrm{min}^{-1}$. L'analyse des résultats montre l'importance des pertes rotoriques dues aux harmoniques de denture, en particulier lorsque la machine est munie d'amortisseurs. Enfin, la comparaison avec des valeurs calculées fait apparaître les limites de la modélisation thermique proposée.
\end{abstract}

\begin{abstract}
The control of synchronous machines by means of static convertors involves an increase of eddycurrents. In this work, the rotor losses are derived from transient temperature rise curves. The tested machine has a solid rotor and four salient poles. The rating is $10 \mathrm{kVA}$ at $3000 \mathrm{r} . \mathrm{p} . \mathrm{m}$. In the experiments, the motor is fed by a current inverter with a constant voltage-frequency ratio. For speeds higher than 200 r.p.m., the machine voltage is used to commutate the inverter. The results show the influence of the armature teeth in rotor losses, particularly for measurements with damper windings. Finally, numerical calculations of eddycurrents losses are achieved. The poor agreement with the results predicted from the thermal analysis exhibit the difficulty to assign reliable values for the thermal constants.
\end{abstract}

\section{Introduction.}

Le développement de l'électronique de puissance a permis l'extension du domaine d'utilisation des moteurs à courant alternatif, car les fonctionnements à vitesses variables ne sont plus réservés aux moteurs à courant continu. L'avantage des machines à courant alternatif est de ne pas avoir de collecteur, ce qui leur permet, en particulier, d'être utilisables en atmosphère explosible et leur confère une puissance massique plus élevée [1]. Par ailleurs, il est possible, grâce à une électronique adaptée, de réaliser différentes fonctions spécifiques comme, par exemple, un fonctionnement à couple constant sur une grande plage de vitesse.

Le moteur synchrone s'adapte très bien à ce genre d'utilisation. En particulier, son absence de glissement permet d'obtenir, d'une manière très précise, des vitesses données. De plus, la présence d'une force électromotrice autorise l'emploi de convertisseurs statiques à commutation naturelle commandée par la machine, ce qui simplifie notablement l'électronique de commande.

Cependant, l'utilisation d'éléments semiconducteurs en régime de commutation introduit des distor- sions de courant et/ou de tension. Il en résulte la présence d'harmoniques qui affectent le champ magnétique de réaction d'induit de la machine. Cela se traduit, au rotor, par un accroissement des pertes électromagnétiques et, en conséquence, des échauffements.

Cet article est relatif aux pertes et échauffements rotoriques d'un moteur synchrone alimenté par un convertisseur statique autopiloté, utilisé en commutateur de courant. La machine testée est à rotor massif et à pôles saillants. Les pertes sont déduites, à l'aide d'un modèle thermique simplifié, de relevés de température effectués en différents points du rotor. Une seconde série d'essais, effectués après cuivrage des calottes polaires, permet d'analyser l'influence d'un amortisseur. En conclusion, on s'efforce de séparer les pertes rotoriques selon leur origine et de les corréler à des prédéterminations effectuées à partir de deux modèles différents.

\section{Dispositifs expérimentaux.}

2.1 CARACTÉRISTIOUES DE LA MACHINE SYNCHRONE TESTÉE. - La machine construite par le 
Centre d'Etudes et de Recherches de la CEM, est tétrapolaire et possède un rotor massif. Ses principales caractéristiques nominales sont :

- Vitesse de rotation $(N): 0$ à $3000 \mathrm{tr} . \mathrm{min}^{-1}$

- Tension simple $\left(V_{\mathrm{n}}\right): 180 \mathrm{~V}$ pour $N=$ $3000 \mathrm{tr} \cdot \mathrm{min}^{-1}$

- Courant d'excitation $\left(I_{\mathrm{fn}}\right): 16 \mathrm{~A}$

- Courant de phase $\left(J_{\mathrm{n}}\right): 19 \mathrm{~A}$

- Puissance apparente $\left(S_{\mathrm{n}}\right): 10 \mathrm{kVA}$ pour $N=3000 \mathrm{tr}$. $\mathrm{min}^{-1}$.

Le stator a comme dimensions principales :

— diamètre : $270 \mathrm{~mm}$

- alésage intérieur : $178 \mathrm{~mm}$

- longueur axiale : $130 \mathrm{~mm}$

- pas polaire : $139,8 \mathrm{~mm}$.

Une coupe du rotor est représentée sur la figure 1. La distance entre deux surfaces polaires opposées est de $174 \mathrm{~mm}$ dans l'axe polaire, ce qui correspond à un entrefer minimal de $2 \mathrm{~mm}$. Après une première série d'essais, une couche de cuivre, d'une épaisseur de $0,6 \mathrm{~mm}$, a été déposée électrolytiquement sur les calottes polaires afin de renforcer l'effet amortisseur du rotor massif.

Le refroidissement de la machine est assuré par convection forcée.

La résistance, mesurée à froid, d'une phase statorique est $R_{\mathrm{a}}=85 \times 10^{-3} \Omega$ et celle de l'inducteur, $R_{\mathrm{f}}=1,34 \Omega$. Un essai de glissement, effectué à une

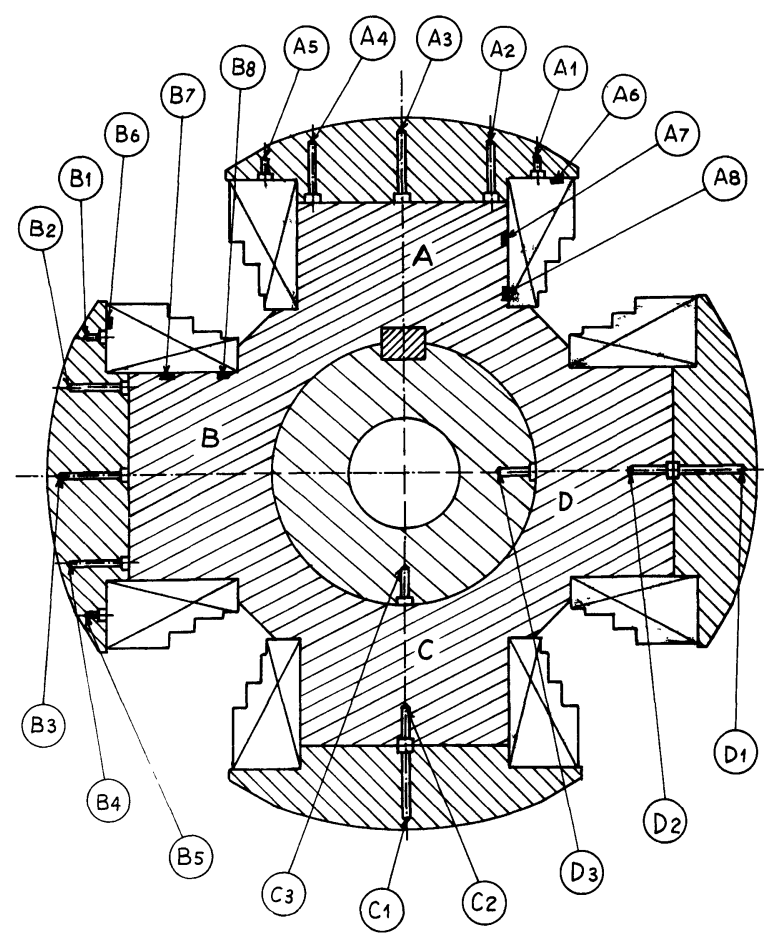

Fig. 1. - Coupe transversale du rotor et implantation des thermocouples $\left(\mathrm{A}_{1}\right.$ à $\mathrm{A}_{8}, \mathrm{~B}_{1}$ à $\mathrm{B}_{8}, \mathrm{C}_{1}$ à $\mathrm{C}_{3}, \mathrm{D}_{1}$ à $\left.\mathrm{D}_{3}\right)$.

[Cross-section of the rotor and location of thermocouple.] vitesse proche de $1500 \mathrm{tr} . \mathrm{min}^{-1}$, conduit aux valeurs d'inductances synchrones d'axes $d$ et $q$ :

$$
L_{d}=5,4 \mathrm{mH} ; L_{q}=3,5 \mathrm{mH} .
$$

L'inductance transitoire, déduite d'un essai en courtcircuit équilibré brusque, vaut $L_{d}^{\prime} \# 1 \mathrm{mH}$. Sa valeur est relativement peu sensible à la présence d'une couche de cuivre sur les surfaces polaires. Enfin, le caractère massif du rotor ne permet pas de déterminer précisément l'inductance subtransitoire d'axe direct [2].

Les caractéristiques à vide $E\left(I_{\mathrm{f}}\right)$, relevées à 480 , 750 et $1500 \mathrm{tr} \cdot \mathrm{min}^{-1}$, sont représentées sur la figure 2. L'absence de coudes de saturation prononcés traduit la faible perméabilité maximale du matériau utilisé pour réaliser les calottes polaires $\left(\mu_{\mathrm{r}} \# 800\right)$. Cela explique également les faibles valeurs des inductances synchrones $L_{d}$ et $L_{q}$.

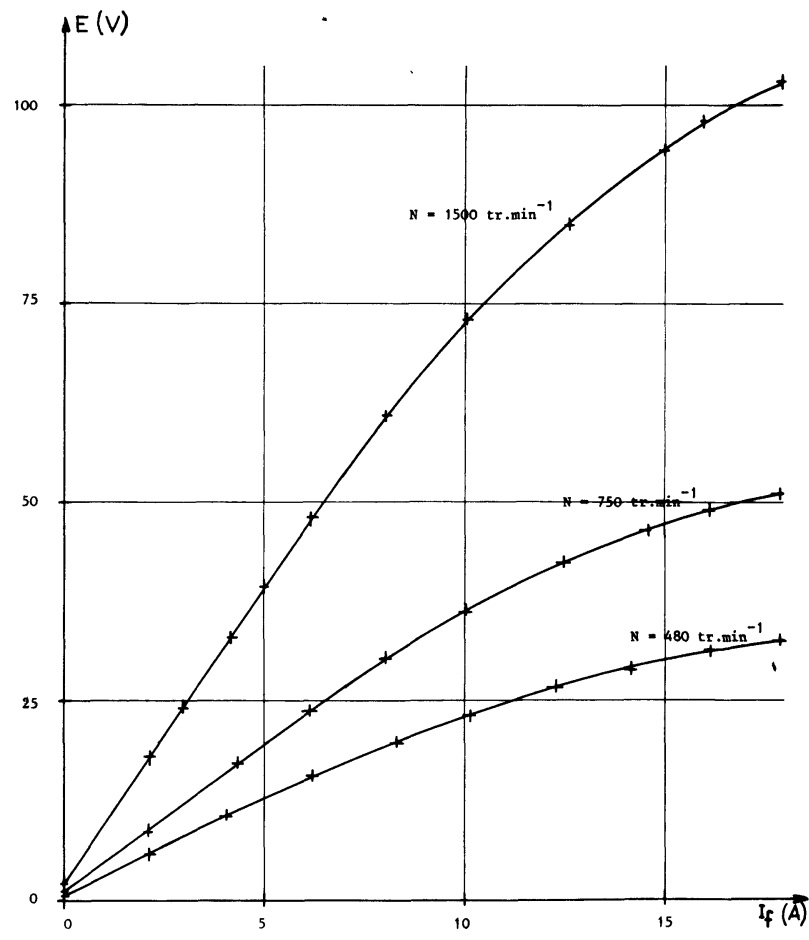

Fig. 2. - Caractéristiques à vide $E=f\left(I_{\mathrm{f}}\right)$.

[No-load phase e.m.f. versus the excitating current $I_{\mathrm{f}}$ ]

2.2 Alimentation DU MOTEUR. - La figure 3 représente le schéma de principe de l'alimentation, constitué de deux ponts de Graetz triphasés à thyristors, la liaison entre les deux étages étant assurée par l'intermédiaire d'une inductance de lissage $L$.

Le réglage du courant $I_{c}$ est effectué par la commande de l'angle de retard à l'amorçage des thyristors du pont PR. Le convertisseur PM, fonc- 


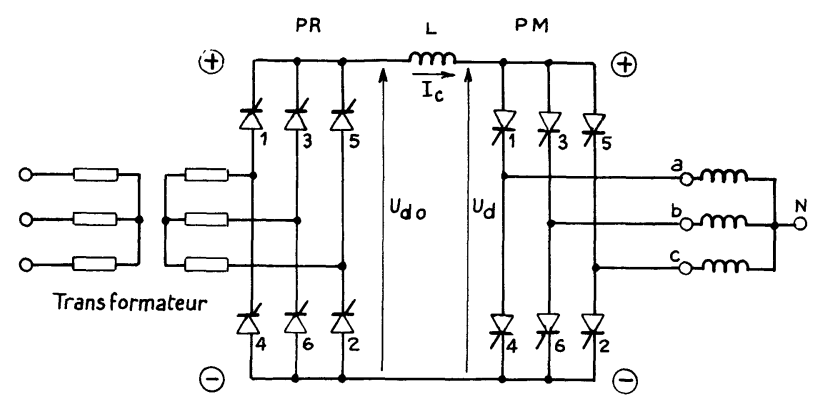

Fig. 3. - Schéma de principe du commutateur de courant.

[Block diagram of the current inverter.]

tionnant en commutateur de courant, est piloté par le moteur synchrone. Un exemple des courants de phases obtenus est donné sur la figure 4. A l'arrêt et aux basses vitesses, les commutations naturelles des thyristors de l'onduleur ne sont pas assurées. Dans ce cas, des circuits auxiliaires sont mis en service afin d'assurer la commutation forcée. Le passage de la phase de démarrage, fonctionnement à fréquence imposée, au mode autopiloté s'effectue automatiquement dès que la machine atteint une vitesse suffisante, fonction des réglages de seuil. Dans ce travail, ne désirant pas explorer le domaine des très basses vitesses, nous avons fixer ce passage à environ $200 \mathrm{tr} \cdot \mathrm{min}^{-1}$.

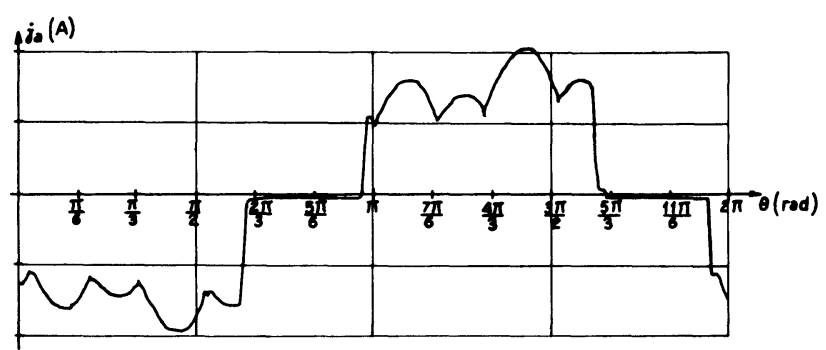

Fig. 4. - Diagramme de courant dans une phase du moteur autopiloté $\left(N=750 \mathrm{tr} \cdot \min ^{-1} ; V_{\mathrm{a}}=47,3 \mathrm{~V}\right.$; $J_{\mathrm{a}}=12$ A).

[Armature current pattern.]

L'alimentation de l'inducteur est prévue pour maintenir un flux magnétique constant, tant que la tension de phase et le courant d'excitation n'ont pas atteint leur valeur nominale. Dans ce but, on utilise un pont mixte dont la commande permet l'asservissement aux grandeurs de consigne.

Les circuits de commande et de régulation sont classiques et ne seront donc pas décrits ici.

2.3 MESURES DES TEMPÉRATURES ROTORIQUES. - Les températures rotoriques sont relevées à l'aide de thermocouples cuivre-constantan.
L'implantation des soudures chaudes est donnée sur la figure 1 ; celles-ci sont situées dans le plan médian du rotor. Les faibles signaux délivrés par les thermocouples nécessitent une amplification et une mise en forme avant transmission par contacts glissants aux appareils de mesures. En effet, le rapport signal/bruit associé à une transmission directe serait trop faible. Cela implique donc l'utilisation de convertisseurs électroniques montés sur des cartes à circuits imprimés, solidaires de l'arbre moteur. Le système utilisé est décrit en détail dans la référence [3]. Le principe consiste à amplifier les signaux délivrés par les thermocouples, puis à les appliquer à un multiplexeur qui transmet les données sélectionnées à un convertisseur tension-fréquence. Celui-ci délivre un signal modulé de $1 \mathrm{~Hz} \cdot \mathrm{mV}^{-1}$ appliqué à deux contacts glissants, puis au fréquencemètre de mesure. L'ensemble est piloté par un microcalculateur qui sélectionne les voies de mesure et traite les données transmises. La précision obtenue est estimée à $\pm 1 \mathrm{~K}$.

Les températures sont exprimées par rapport à celle des soudures froides, fixées sur le circuit convertisseur de mesures.

Le stator est équipé de 4 thermocouples implantés sur l'alésage intérieur.

\subsection{ANALYSE DES COURANTS STATORIQUES.} - Les pertes rotoriques dépendent en grande partie du champ de réaction d'induit et donc de la forme des courants de phase. Aussi, pour chaque essai, une analyse harmonique des courants est effectuée. Dans ce but, une courbe de courant, visualisée sur une période, est mémorisée dans un oscilloscope à mémoires numériques, puis transférée à un microcalculateur. Ce dernier réalise l'analyse harmonique à l'aide d'un programme de transformée de Fourier rapide d'après l'algorithme de Cooley-Turkey [4]. Pour bénéficier d'un compromis acceptable entre le temps de calcul et une précision correcte (erreur relative inférieure à $2,5 \%$ ), l'acquisition s'effectue par un échantillonnage en 512 points.

\section{Résultats.}

Les relevés d'échauffement correspondent à des essais effectués en alternateur à vide et en moteur autopiloté, à des vitesses de 480,750 et $1500 \mathrm{tr} \cdot \mathrm{min}^{-1}$ et pour un courant d'excitation égal à la valeur nominale $\left(I_{\mathrm{fn}}=16 \mathrm{~A}\right)$. En charge, le courant de phase est réglé à environ $12 \mathrm{~A}$.

La vitesse maximale a été limitée à $1500 \mathrm{tr} . \mathrm{min}^{-1}$ pour deux raisons. La première est due aux échauffements qui, lorsque les surfaces polaires sont cuivrées, deviennent rapidement prohibitifs avec la vitesse. La seconde est liée aux problèmes de tenue mécanique des composants montés sur les cartes à circuits imprimés, solidaires de l'arbre moteur ; une meilleure implantation aurait permis de lever cet écueil. 
Les mesures montrent que les écarts de températures au sein de la roue polaire sont suffisamment faibles $(<3 \mathrm{~K})$ pour qu'en première approximation, celle-ci puisse être considérée comme un solide homogène. Le gradient thermique est essentiellement localisé entre la roue polaire et le bobinage inducteur. En conséquence, la modélisation thermique du rotor est basée sur un modèle à 2 zones :

- la roue polaire, de température moyenne $T_{\mathrm{r}}$, dans laquelle sont dissipées les pertes électromagnétiques $p_{\mathrm{r}}$, objets de l'étude ;

- l'inducteur, de température moyenne $T_{\mathrm{cu}}$, pour lequel les pertes $p_{\text {cu }}$ sont calculables.

Dans un but de clarté, seules les températures moyennes $T_{\mathrm{r}}$ et $T_{\mathrm{cu}}$ seront données ici.

Le schéma simplifié de la figure 5 indique les conditions de l'écoulement de l'air dans le moteur. Celui-ci, en provenance du ventilateur, circule dans l'entrefer et dans les espaces interpolaires avant d'être rejeté par les évents d'aération. Les échanges par convection se font aux niveaux des surfaces polaires en ce qui concerne la roue polaire et aux niveaux des espaces interpolaires et des zones frontales avant et arrière en ce qui concerne le bobinage inducteur. Dans le modèle proposé, le couplage thermique entre le rotor et le stator est négligé. Nous avons tenté d'évaluer l'erreur alors commise en effectuant des mesures à l'arrêt. Dans ce but, seules deux phases de l'induit sont alimentées en courant continu. Les relevés montrent que le bobinage inducteur et la roue polaire sont à la même température et que le couplage thermique entre le rotor et le stator correspond à environ $5 \%$ de la puissance transférée à travers les surfaces polaires. Dans le cadre des approximations faites, cette erreur nous semble acceptable.

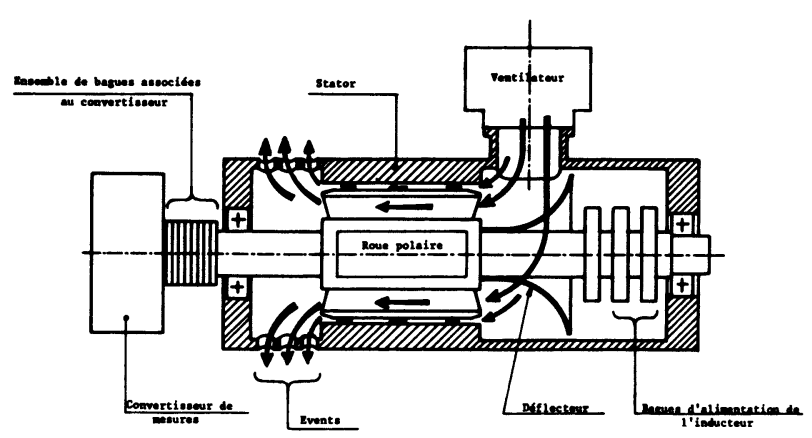

Fig. 5. - Coupe schématique de la machine.

[Schematic cross-section of the synchronous machine.]

Dans ces conditions, l'équation d'évolution, reliant les pertes électromagnétiques $p_{\mathrm{r}}$ et les températures $T_{\mathrm{r}}$ et $T_{\mathrm{cu}}$, s'écrit [5] :

$$
p_{\mathrm{r}}=C_{\mathrm{r}} \frac{\mathrm{d} T_{\mathrm{r}}}{\mathrm{d} t}+H_{\mathrm{r}} T_{\mathrm{r}}+k\left(T_{\mathrm{r}}-T_{\text {cu }}\right)
$$

où $C_{\mathrm{r}}$ est la capacité thermique de la roue polaire, $H_{\mathrm{r}}$ le coefficient d'échange par convection entre les surfaces polaires et l'entrefer et $k$ le coefficient d'échange par conduction entre la roue polaire et l'inducteur. Les températures sont exprimées par rapport à celle du milieu ambiant avec lequel s'effectuent les échanges convectifs. Une équation analogue à la relation (1) s'applique à l'inducteur. En supposant les coefficients d'échanges thermiques indépendants de la température, les échauffements $T_{\mathrm{r}}$ et $T_{\mathrm{cu}}$ évoluent au cours du temps selon les lois de la forme :

$$
\begin{gathered}
T_{\mathrm{r}}=\left(T_{\mathrm{r}}\right)_{\mathrm{f}}+A_{\mathrm{r}} \mathrm{e}^{-\frac{t}{\tau_{1}}}+B_{\mathrm{r}} \mathrm{e}^{-\frac{t}{\tau_{2}}} \\
T_{\text {cu }}=\left(T_{\text {cu }}\right)_{\mathrm{f}}+A_{\text {cu }} \mathrm{e}^{-\frac{t}{\tau_{1}}}+B_{\text {cu }} \mathrm{e}^{-\frac{t}{\tau_{2}}} .
\end{gathered}
$$

Le couplage thermique entre les deux zones entraîne que les constantes de temps $\tau_{1}$ et $\tau_{2}$ sont respectivement différentes des constantes $\tau_{\mathrm{r}}$ et $\tau_{\mathrm{cu}}$, associées à chaque zone supposée évoluer séparément. Un calcul approximatif de $\tau_{1}$ et $\tau_{2}$ montre que leurs valeurs respectives sont notablement différentes $\left(\tau_{1} \gg \tau_{2}\right)$. Cela permet de les déterminer par une méthode graphique. En effet, dès que $t \gg \tau_{2}$, les variations de $T_{\mathrm{r}}$ et $T_{\mathrm{cu}}$ sont données par :

$$
\begin{gathered}
T_{\mathrm{r}}-\left(T_{\mathrm{r}}\right)_{\mathrm{f}}=A_{\mathrm{r}} \mathrm{e}^{-\frac{t}{\tau_{1}}} \\
T_{\mathrm{cu}}-\left(T_{\mathrm{cu}}\right)_{\mathrm{f}}=A_{\mathrm{cu}} \mathrm{e}^{-\frac{t}{\tau_{1}}}
\end{gathered}
$$

ce qui correspond, en coordonnées semi-logarithmiques, à des droites de pente $-\frac{1}{\tau_{1}}$. La constante $\tau_{2}$ est obtenue en considérant les écarts entre les évolutions mesurées et les extrapolations, aux faibles valeurs de $t$, des droites précédemment définies.

Un exemple des échauffements obtenus à $1500 \mathrm{tr} \cdot \mathrm{min}^{-1}$ est donné sur les figures 6 et 7. Ceux-ci correspondent à des essais effectués avant et après cuivrage des calottes polaires. On constate que le cuivrage entraîne une augmentation des échauffements rotoriques. De plus, les gradients thermiques s'inversent, la température de la roue polaire devenant supérieure à celle de l'inducteur. La détermination graphique des constances de temps $\tau_{1}$ et $\tau_{2}$, non représentée ici, montre que leur valeur n'est pas affectée par le cuivrage des surfaces polaires. Cela signifie que les échanges convectifs ne sont pas sensiblement modifiés du fait de la présence de la couche de cuivre. Par contre, ces constantes dépendent de la vitesse de rotation, ce qui montre que les échanges convectifs ne sont pas imposés uniquement par la ventilation forcée. Il faut enfin noter que la détermination de $\tau_{2}$ est très imprécise du fait de sa 


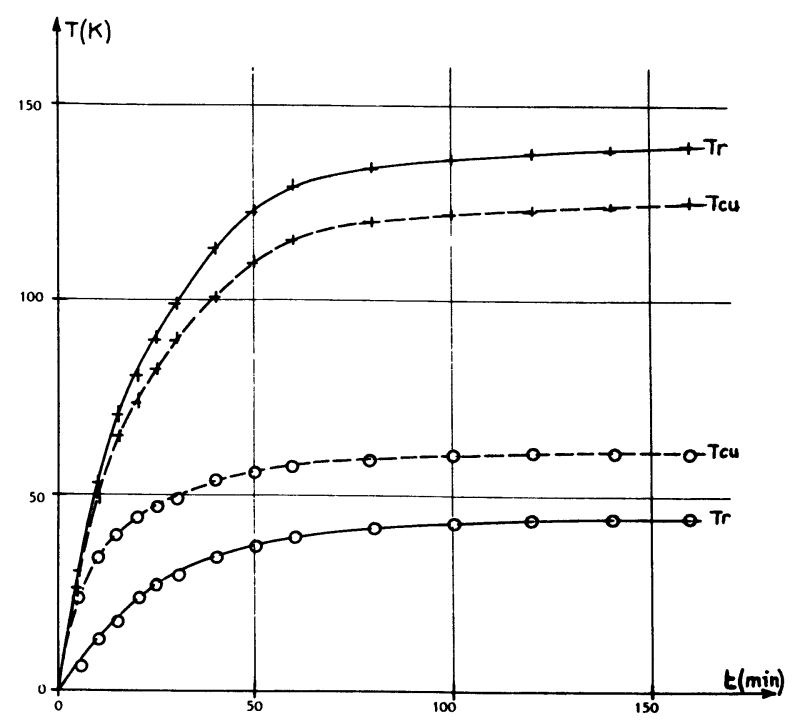

Fig. 6. - Echauffements rotoriques lors d'essais en alternateur à vide $\left(N=1500 \mathrm{tr} . \mathrm{min}^{-1} ; I_{\mathrm{f}}=16 \mathrm{~A}\right)$. $-T_{\mathrm{r}}$ (roue polaire) avant cuivrage ; ---O--- $T_{\mathrm{cu}}$ (inducteur) avant cuivrage ; $-+T_{\mathrm{r}}$ après cuivrage ;

------ $T_{\text {cu }}$ après cuivrage.

[Temperature-rises of the rotor in no-load runs.]

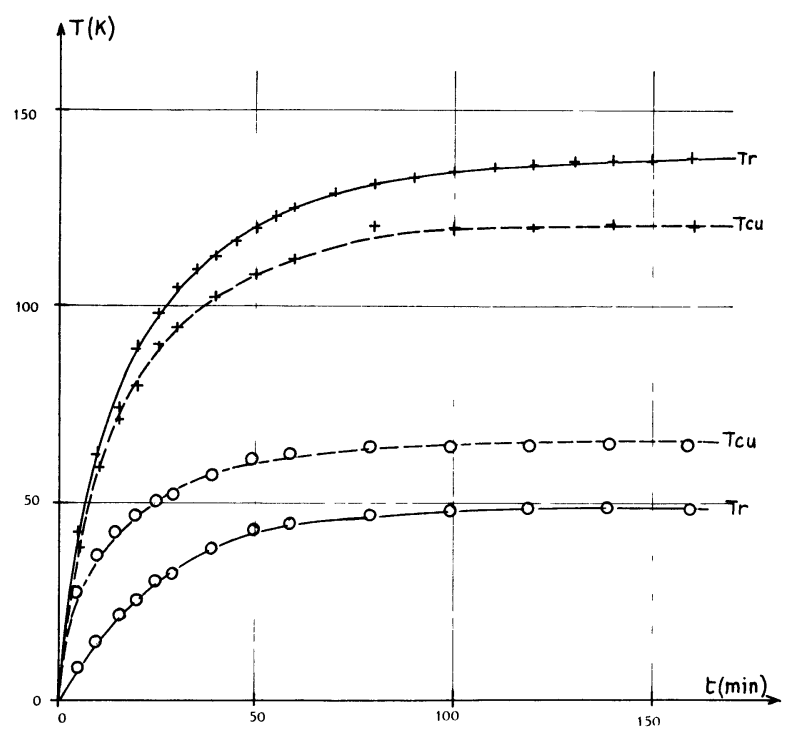

Fig. 7. - Echauffements en moteur autopiloté $(N=$ $\left.1500 \mathrm{tr} . \min ^{-1} ; I_{\mathrm{f}}=16 \mathrm{~A} ; J_{\mathrm{a}}=12 \mathrm{~A}\right)$. O relevés avant cuivrage ; + relevés après cuivrage.

[Temperature-rises in the self-controlled synchronous machine.]

faible valeur $\left(\tau_{2} \# 4 \mathrm{~min}\right)$ comparée à celle de $\tau_{1}$ (environ $30 \mathrm{~min}$ ). Malgré ses imperfections, la modélisation thermique proposée permet, dans une première approche, de décrire les phénomènes thermiques développés au rotor.
Les pertes $p_{\mathrm{r}}$, dissipées dans la roue polaire, sont déduites des relevés d'échauffement en régime stationnaire. Dans ces conditions, l'équation (1) s'écrit :

$$
p_{\mathrm{r}}=H_{\mathrm{r}}\left(T_{\mathrm{r}}\right)_{\mathrm{f}}+k\left(T_{\mathrm{r}}-T_{\mathrm{cu}}\right)_{\mathrm{f}} \text {. }
$$

La résolution de cette équation nécessite la connaissance préalable de $H_{\mathrm{r}}$ et $k$. Ces deux coefficients sont déterminés à l'aide d'essais complémentaires, en supposant que $H_{\mathrm{r}}$ ne dépend que de la vitesse de rotation et que $k$ est une constante pour tous les essais. Les deux mesures complémentaires sont effectuées dans les conditions suivantes :

a) Machine à l'arrêt, l'inducteur étant parcouru par un courant $I_{\mathrm{f}}=16 \mathrm{~A}$. Les seules pertes sont celles dissipées par effet Joule dans l'inducteur $\left(p_{\mathrm{cu}}\right)$.

b) Machine entraînée à vitesse imposée ( 1500 , 750,480 et 0 tr $\cdot \min ^{-1}$ ), deux phases de l'induit, mises en série, étant alimentées par un courant sinusoïdal de fréquence $50 \mathrm{~Hz}$, et de valauer efficace 16 A. Durant ces essais, l'inducteur est ouvert $\left(I_{\mathrm{f}}=0\right)$. En supposant que les pertes statoriques sont faibles devant celles dissipées au rotor, ces dernières sont égales à la somme de la puissance électrique fournie à l'induit et de la puissance mécanique, déduite de la valeur du couple d'entraînement.

Ainsi, la connaissance simultanée de $p_{\mathrm{r}}$ et des températures d'équilibre, permet, à l'aide de l'équation (4), d'écrire un système conduisant aux valeurs de $k$ et $H_{\mathrm{r}}$ cherchées. Les résultats obtenus sont consignés dans le tableau I. Sur ce tableau sont également portées les valeurs de la constante de temps $\tau_{1}$, déterminées comme il est indiqué précédemment, et du produit $\tau_{1} H_{\mathrm{r}}$.

Le tableau I fait apparaître que le coefficient d'échange par convection $H_{\mathrm{r}}$ augmente avec la vitesse alors que $\tau_{1}$ diminue, le produit des deux coefficients, égale en première approximation à la capacité thermique de la roue polaire, restant sensiblement constant.

Les pertes rotoriques déduites des mesures d'échauffement apparaissent dans le tableau II. Afin de caractériser les essais, les valeurs efficaces du courant et de la tension de phase ainsi que la puissance utile et le facteur de puissance sont également notées. Les notations $\mathrm{AV} \mathrm{Cu}$ et $\mathrm{AP} \mathrm{Cu}$ désignent les essais réalisés respectivement avant et après le cuivrage des pôles inducteurs. L'examen du tableau montre que les pertes rotoriques en alternateur à vide ne sont pas négligeables devant celles correspondant au fonctionnement en moteur autopiloté. De plus, il apparaît que ces pertes augmentent fortement du fait de la présence d'une couche de cuivre sur les surfaces polaires. Ce dernier point explique l'inversion des gradients thermiques observés sur les figures 6 et 7 . 
Tableau I. - Coefficients d'échanges thermiques.

\begin{tabular}{|c|c|c|c|c|}
\hline $\begin{array}{c}N \\
\left(\operatorname{tr} \min ^{-1}\right)\end{array}$ & $\begin{array}{c}k \\
\left(\mathrm{~W} . \mathrm{K}^{-1}\right)\end{array}$ & $\begin{array}{c}H_{\mathrm{r}} \\
\left(\mathrm{W} . \mathrm{K}^{-1}\right)\end{array}$ & $\begin{array}{c}\tau_{1} \\
\left(10^{3} \mathrm{~s}\right)\end{array}$ & $\begin{array}{c}\tau_{1} H_{\mathrm{r}} \\
\left(10^{3} \mathrm{~J} . \mathrm{K}^{-1}\right)\end{array}$ \\
\hline 0 & 11,6 & 4,33 & & \\
\hline 480 & 11,6 & 4,74 & $2,14 \pm 0,08$ & 10,1 \\
\hline 750 & 11,6 & 5,31 & $2,00 \pm 0,04$ & 10,6 \\
\hline 1500 & 11,6 & 7,26 & $1,60 \pm 0,05$ & 11,6 \\
\hline
\end{tabular}

\section{Discussion.}

Les pertes dissipées dans la roue polaire, essentiellement liées aux courants de Foucault induits, ont deux origines [6] :

- les harmoniques créés par la variation périodique de la perméance d'entrefer, liée à la présence des dents et des encoches de l'alésage du stator, appelés par la suite harmoniques de denture,

- les harmoniques créés par les champs tournants de réaction d'induit associés aux harmoniques d'espace et de temps des f.m.m. statoriques.
Dans les essais en alternateur à vide, les pertes $p_{\mathrm{r}}$ sont dues uniquement aux harmoniques de denture. En ce qui concerne le fonctionnement en moteur autopiloté, une méthode pour séparer les pertes consiste à admettre que les pertes par harmoniques de denture ne dépendent que de la vitesse de rotation et du flux résultant. Si on néglige les chutes de tension dues à la résistance de l'induit et à l'inductance de fuites, la f.e.m. résultante correspond à la tension aux bornes de la machine. L'examen du tableau II montre que $V_{\mathrm{a}}$ varie légèrement entre les essais en alternateur à vide et en

Tableau II. - Pertes rotoriques déduites des échauffements du rotor.

\begin{tabular}{|c|c|c|c|c|c|c|c|}
\hline \multicolumn{3}{|c|}{$I_{\mathrm{f}}=16 \mathrm{~A}$} & \multirow{2}{*}{$\begin{array}{l}V_{\mathrm{a}} \\
(\mathrm{V})\end{array}$} & \multirow{2}{*}{$\begin{array}{c}J_{\mathbf{a}} \\
\text { (A) }\end{array}$} & \multirow{2}{*}{$\begin{array}{c}P_{\mathrm{u}} \\
(\mathrm{W})\end{array}$} & \multirow{2}{*}{$\lambda$} & \multirow{2}{*}{$\begin{array}{c}P_{\mathrm{r}} \\
(\mathrm{W})\end{array}$} \\
\hline$N\left(\operatorname{tr} \min ^{-1}\right)$ & & ssai & & & & & \\
\hline \multirow{4}{*}{480} & \multirow{2}{*}{$\begin{array}{l}\text { Alter- } \\
\text { nateur } \\
\text { à vide }\end{array}$} & $\mathrm{AV} \mathrm{Cu}$ & 30,1 & 0 & 0 & & 28 \\
\hline & & $\mathrm{AP} \mathrm{Cu}$ & 30,8 & 0 & 0 & & 210 \\
\hline & \multirow{2}{*}{$\begin{array}{l}\text { Moteur } \\
\text { auto-- } \\
\text { piloté }\end{array}$} & $\mathrm{AV} \mathrm{Cu}$ & 30,6 & 11,2 & 709 & 0,82 & 69 \\
\hline & & $\mathrm{AP} \mathrm{Cu}$ & 30,4 & 11,9 & 656 & 0,84 & 260 \\
\hline \multirow{4}{*}{750} & \multirow{2}{*}{$\begin{array}{l}\text { Alter- } \\
\text { nateur } \\
\text { à vide }\end{array}$} & $\mathrm{AV} \mathrm{Cu}$ & 46,5 & 0 & 0 & & 54 \\
\hline & & $\mathrm{AP} \mathrm{Cu}$ & 48,3 & 0 & 0 & & 440 \\
\hline & \multirow{2}{*}{$\begin{array}{l}\text { Moteur } \\
\text { auto-- } \\
\text { piloté }\end{array}$} & $\mathrm{AV} \mathrm{Cu}$ & 47,1 & 12,2 & 1270 & 0,85 & 90 \\
\hline & & $\mathrm{AP} \mathrm{Cu}$ & 47,3 & 12,0 & 1040 & 0,85 & 440 \\
\hline \multirow{4}{*}{1500} & \multirow{2}{*}{$\begin{array}{l}\text { Alter- } \\
\text { nateur } \\
\text { à vide }\end{array}$} & $\mathrm{AV} \mathrm{Cu}$ & 93,7 & 0 & 0 & & 140 \\
\hline & & $\mathrm{AP} \mathrm{Cu}$ & 95,2 & 0 & 0 & & 1200 \\
\hline & \multirow{2}{*}{$\begin{array}{l}\text { Moteur } \\
\text { auto- } \\
\text { piloté }\end{array}$} & $\mathrm{AV} \mathrm{Cu}$ & 93,1 & 12,8 & 2880 & 0,91 & 170 \\
\hline & & $\mathrm{AP} \mathrm{Cu}$ & 92,2 & 12,0 & 1880 & 0,88 & 1200 \\
\hline
\end{tabular}


moteur autopiloté. Ces écarts, relativement faibles, pourraient sembler négligeables. Mais, le relevé des courbes représentant les pertes fer totales en fonction de la f.e.m. à vide, obtenues de façon classique et non représentées ici, montre une forte variation des pertes avec la f.e.m. [7]. Cela est lié à l'augmentation du flux qui provoque la saturation des épaulements des encoches semi-fermées, ce qui accroît la variation de réluctance. Ainsi, la séparation des pertes nécessite de les rapporter à une même tension $V_{\mathrm{a}}$. Cette correction est effectuée en se basant sur les variations des pertes fer totales. Le tableau III résume les valeurs des différentes pertes dissipées au rotor lors du fonctionnement en moteur autopiloté. Le terme $p_{\text {hd }}$ représente les pertes en alternateur à vide, ramenées à la tension $V_{\mathrm{a}}$ indiquée ; la différence entre $p_{\mathrm{r}}$ et $p_{\text {hd }}$ donne les pertes $p_{\mathrm{hc}}$, associées aux harmoniques de courant. Sur ce tableau, les premiers harmoniques de courant (de rang 5 et 7) sont caractérisés par une grandeur $J_{6}$ définie par la relation :

$$
J_{6}=\sqrt{J_{5}^{2}+J_{7}^{2}}
$$

où $J_{5}$ et $J_{7}$ représentent respectivement les valeurs efficaces des harmoniques 5 et 7 , tous deux responsables de courants de Foucault de même pulsation $6 \omega$ au rotor.

Précisons que la séparation des pertes ainsi effectuée est discutable. En effet, dans un fonctionnement en charge, la réaction magnétique d'induit transversale entraîne, à flux identique, des variations de réluctance dues à la denture statorique différentes de celles associées à un fonctionnement à vide. Il s'ensuit que l'estimation de $p_{\text {hd }}$ est incertaine. Dans notre cas, l'erreur commise doit cependant être limitée, du fait de la faible valeur relative de la réaction magnétique d'induit.

Tableau III. - Séparation des pertes dans le cas du fonctionnement en moteur autopiloté.

\begin{tabular}{|c|c|c|c|c|c|c|c|}
\hline \multicolumn{2}{|c|}{$I_{\mathrm{f}}=16 \mathrm{~A}$} & \multirow{2}{*}{$\begin{array}{c}V_{\mathrm{a}} \\
(\mathrm{V})\end{array}$} & $\begin{array}{c}J_{\mathrm{a}} \\
(\mathrm{A})\end{array}$ & $\begin{array}{c}J_{6} \\
(\mathrm{~A})\end{array}$ & $\begin{array}{c}p_{\mathrm{r}} \\
(\mathrm{W})\end{array}$ & $\begin{array}{c}p_{\mathrm{hd}} \\
(\mathrm{W})\end{array}$ & $\begin{array}{c}p_{\mathrm{hc}} \\
(\mathrm{W})\end{array}$ \\
\hline \multirow{2}{*}{480} & $\mathrm{AV} \mathrm{Cu}$ & 30,6 & 11,2 & 3,2 & 69 & 29 & 40 \\
\cline { 2 - 8 } & $\mathrm{AP} \mathrm{Cu}$ & 30,4 & 11,9 & 2,7 & 260 & 213 & 47 \\
\hline \multirow{3}{*}{750} & $\mathrm{AV} \mathrm{Cu}$ & 47,1 & 12,2 & 3,2 & 90 & 56 & 34 \\
\cline { 2 - 9 } & $\mathrm{AP} \mathrm{Cu}$ & 47,3 & 12 & 3,0 & 440 & 420 & 20 \\
\hline \multirow{3}{*}{1500} & $\mathrm{AV} \mathrm{Cu}$ & 93,1 & 12,8 & 2,8 & 170 & 130 & 40 \\
\cline { 2 - 8 } & $\mathrm{AP} \mathrm{Cu}$ & 92,2 & 12 & 3,0 & 1200 & 1100 & 100 \\
\hline
\end{tabular}

L'examen du tableau III montre que les pertes par harmoniques de denture augmentent avec la vitesse et sont nettement plus importantes après cuivrage qu'avant. En ce qui concerne les pertes par harmoniques de courant, les variations observées n'apparaissent pas clairement corrélées aux conditions expérimentales.

Afin de mieux analyser les résultats des essais, les pertes par courants de Foucault dissipées au rotor ont été prédéterminées à partir de deux modèles.

Le premier est basé sur la résolution des équations de Maxwell dans une géométrie semi-infinie en supposant les matériaux linéaires [8,9]. Avec ces hypothèses simplificatrices, on obtient une expression analytique des pertes. Outre les paramètres géométriques de la machine, les calculs font intervenir la pulsation des courants induits au rotor, l'amplitude de l'harmonique de courant considéré et les caractéristiques du matériau (conductivité $\sigma$ et perméabilité $\mu$ ). La difficulté réside dans la valeur de $\mu$ à utiliser. Ce modèle permet d'obtenir les pertes liées aux harmoniques de courant et celles associées aux harmoniques de denture. Dans ce dernier cas, il est cependant nécessaire de faire intervenir, dans les calculs, un courant fictif à l'origine des pertes. L'état de saturation moyen étant pratiquement identique pour tous les essais, une même valeur arbitraire est choisie. Les résultats obtenus n'ont alors de signification qu'en valeurs relatives.

Le second modèle, plus performant que le précédent, est basé sur la résolution des équations par la méthode des éléments finis [10]. Les temps de calcul, relativement longs, limitent la prédétermination des pertes associées aux seuls harmoniques de courant. De plus, la perméabilité relative utilisée est supposée constante, égale à $\mu_{\mathrm{r}}=50$. Le choix de 


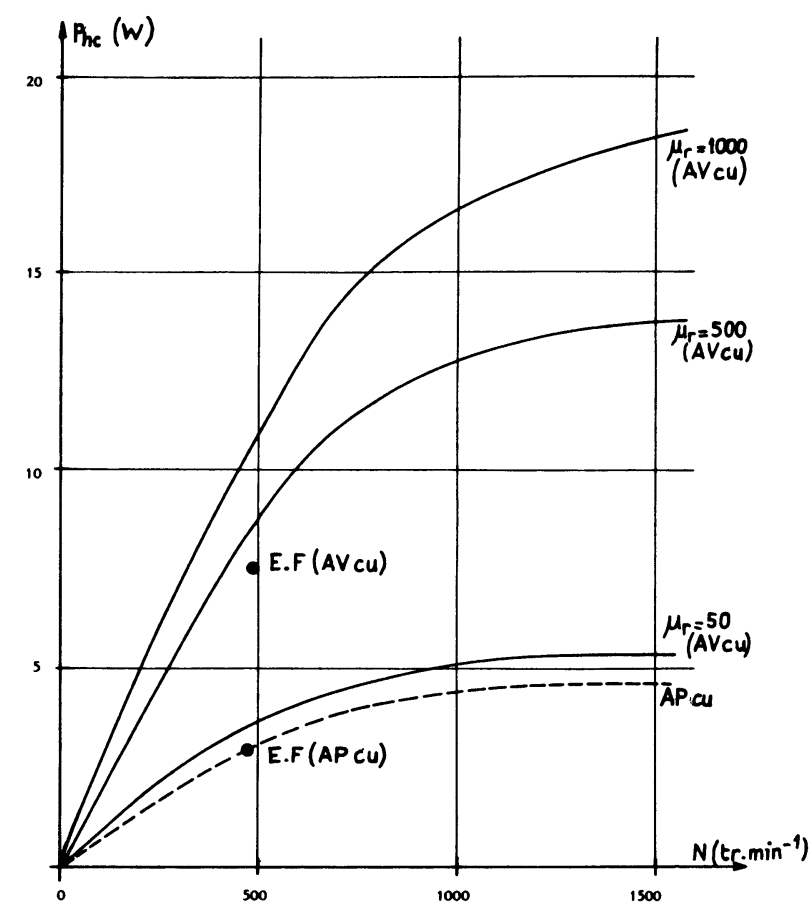

Fig. 8. - Comparaison des pertes rotoriques, dues aux harmoniques de courant, calculées selon un modèle simplifié et par une méthode d'éléments finis. ... modèle simplifié ; - calcul par éléments finis [10].

[Rotor losses induced by harmonic components of armature currents, derived from classical model and finiteelement method.]

cette faible valeur s'explique en notant que les harmoniques concernés entraînent la formation de cycles secondaires, le matériau rotorique étant aimanté du fait de l'existence d'un champ synchrone. La détermination des pertes par harmoniques de denture, de rang 24 , nécessiterait un maillage trop fin rendant la longueur du temps de calcul prohibitif.

Les résultats des calculs sont présentés sur les figures 8 et 9 . La figure 8 est relative aux pertes par harmoniques de courant. Avec le modèle simplifié, les calculs sont effectués en ne tenant compte que des harmoniques de courant de rang 5 et 7 (valeurs mesurées lors des essais) et pour trois valeurs de la perméabilité relative $\left(\mu_{\mathrm{r}}=50,500,1000\right)$. Lorsque les surfaces des pôles sont cuivrées (AP Cu), on obtient des valeurs peu différentes selon $\mu_{r}$ choisi. Cela explique que, dans ce cas, une seule courbe est présentée. Par contre, on observe, en l'absence de cuivrage, une forte variation des pertes selon la valeur de $\mu_{\mathrm{r}}$ utilisée. Le calcul par éléments finis [10] est réalisé en utilisant les paramètres suivants :

$$
\mu_{\mathrm{r}}=50 \quad J_{\mathrm{a}}=20 \mathrm{~A} \quad N=480 \operatorname{tr} \cdot \mathrm{min}^{-1} .
$$

L'onde de courant est supposée idéale (temps de commutation nuls). Ce modèle prend également en compte les harmoniques d'espace. Afin de ramener les valeurs calculées aux conditions des essais, les pertes sont supposées proportionnelles au carré de l'intensité. Les deux valeurs obtenues (AV Cu et AP $\mathrm{Cu}$ ) sont portées sur la figure 8. L'examen de cette figure montre que les pertes calculées sont nettement inférieures à celles déduites des essais thermiques et que le cuivrage s'accompagne d'une diminution des pertes. Dans le cas des essais après cuivrage, il est clair que la précision obtenue est faible, la majeure partie de l'échauffement étant liée aux pertes par harmoniques de denture. En se limitant aux résultats obtenus avant cuivrage, les divergences observées peuvent avoir trois origines :

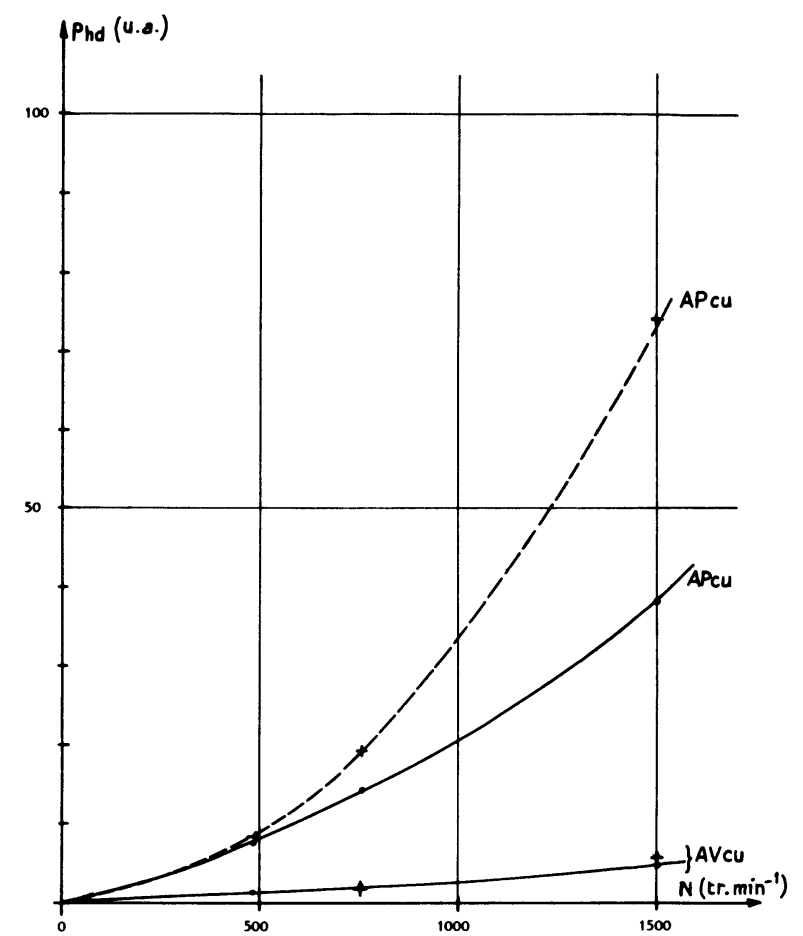

Fig. 9. - Variation relative des pertes par harmoniques de denture en fonction de la vitesse. + calculs selon la méthode simplifiée ; valeurs déduites des essais thermiques.

[Harmonic losses induced by the armature teeth from classical model and thermal analysis (in arbitrary unit).]

- la première est due à l'imprécision sur la valeur des pertes déduites de la modélisation thermique (incertitudes sur les relevés de température, simplification trop poussée du modèle utilisée) ;

- en second lieu, il n'est pas démontré qu'il soit licite, comme nous l'avons supposé, d'écrire que les pertes dissipées dans la roue polaire sont la somme des pertes par harmonique de denture et de celles par harmoniques de courant ;

- enfin, les calculs sont effectués en négligeant les phénomènes d'hystérésis, associés en particulier à des champs d'excitation tournants, qui dépendent de l'état de saturation local du matériau rotorique.

La figure 9 présente, en valeurs relatives, les pertes par harmoniques de denture, calculées par la méthode simplifiée et déduites des essais d'échauffement. Dans les deux cas, les pertes dissipées, en 
l'absence de cuivre, à $480 \mathrm{tr} \cdot \mathrm{min}^{-1}$ sont prises arbitrairement égales à 1 . Dans les calculs, la perméabilité relative du matériau est supposée constante et égale à 50 . Les courbes calculées et déduites des essais montrent que le cuivrage des pôles s'accompagne d'une augmentation notable des pertes, dissipées essentiellement dans la couche de cuivre. Ce résultat est conforme à celui décrit dans la référence [8], où il est par ailleurs montré qu'il existe, dans certains cas, une épaisseur optimale de la couche de cuivre permettant de diminuer à la fois les pertes par harmoniques de courant et par harmoniques de denture. Dans le cas de la machine testée, les calculs effectués pour des couches de cuivre variant entre 0 et $1,5 \mathrm{~mm}$ montrent que les pertes par harmoniques de denture augmentent de façon monotone avec l'épaisseur.

\section{Conclusion.}

Cette étude fait apparaître l'intérêt et les limites de l'analyse thermique en ce qui concerne la détermination et la séparation des pertes rotoriques dans une machine tournante. Il existe peu de méthodes pour les atteindre, et des relevés de températures, associés à une modélisation thermique, en permettent l'analyse. Cependant, ce travail et d'autres, effectués, par exemple, sur des machines asynchrones [11-13], montrent que la précision concernant la détermination des pertes, et leur répartition, est directement liée à celle des relevés de température et à la finesse du modèle thermique utilisé. Ainsi, une précision de $\pm 0,1 \mathrm{~K}$ sur les échauffements semble nécessaire (au lieu de $\pm 1 \mathrm{~K}$ obtenue dans nos essais). Cela pose évidemment le problème des capteurs et de la transmission des données.

De façon au moins qualitative, les essais indiquent que si les harmoniques de courant associés au mode d'alimentation du moteur créent des pertes rotoriques, ces dernières ne sont jamais prépondérantes devant celles induites par les harmoniques de denture. Ce résultat valable dans le cas d'une machine à rotor massif, ne doit évidemment pas être étendu, sans précaution, aux machines à rotor feuilleté.

Actuellement, nous tentons de mieux préciser les pertes en abordant le problème de manière différente. L'approche consiste en la mesure des inductances harmoniques, leur partie imaginaire rendant compte des pertes en régime asynchrone. Du fait de la méthode de mesure de ces inductances [14], il est clair que seules les pertes associées au champ de réaction d'induit peuvent être obtenues. Leur détermination précise est néanmoins un point essentiel du fait du manque de données en ce qui concerne le comportement des matériaux magnétiques soumis à des champs tournants. En conséquence, la prédétermination des pertes dans les machines associées à des convertisseurs statiques est encore hasardeuse.

\section{Bibliographie}

[1] ANDERS, E. K., Entraînements à courant alternatif à vitesse variable pour environnements sévères. Revue ASEA 3 (1978).

[2] RAZEK, A. A., Natural commutation in solid rotor synchronous machines associated with static convectors, Electr. Mach. Electromech. 4 (1979) 331.

[3] Milimonfared, D., Contribution à la modélisation des machines synchrones à rotor massif et à pôles saillants. Applications aux moteurs autopilotés. Thèse de Docteur-Ingénieur, Université P. et M. Curie (1984).

[4] Cooley, J. W. et Tukey, J. W., An algorithm for the machine calculation of complex Fourier series. Math. Comput. 19 (1965) 297.

[5] KREITH, Transmission de chaleur et thermodynamique (Masson éd., Paris) 1967.

[6] Lechat, D., Rialland, J. F., Bonnefille, R., Analyse des pertes rotoriques dissipées dans les machines synchrones associées à des convertisseurs statiques, Revue Phys. Appl. 19 (1984) 395.

[7] PARChemin, M., Contribution à l'étude des pertes rotoriques et de la commutation des éléments semiconducteurs dans un moteur synchrone autopiloté, Mémoire d'Ingénieur CNAM, Paris (1985).
[8] Darnand H. et Grellet, G., Pole face losses for synchronous machines with solid salient copperfaced shoes and fed by no sinusoidal current waves, Proc. ICEM, Budapest, 853 (1982).

[9] Portier, A., Programme de calcul des pertes superficielles d'un rotor. Document C.E.M. FXLL/84O28.

[10] Bouillault, F., Contribution à la modélisation de systèmes électromagnétiques en mouvement comportant des parties ferromagnétiques massives. Thèse $3^{\mathrm{e}}$ cycle, Université $\mathbf{P}$. et $\mathbf{M}$. Curie (1984).

[11] BAHBOUTH, S., Etude en régime permanent d'une machine asynchrone alimentée par un onduleur à transistors. Performances, pertes, échauffements. Thèse de Docteur-Ingénieur, INP de Grenoble (1981).

[12] NAMburi, N. R. et BARTON, T. H., Thermal modelling of an induction motor, IEEE PAS-102, 8 (1983) 2636.

[13] ROYE, D., Modélisation thermique des machines électriques tournantes. Application à la machine à induction, Thèse de Doctorat es-Sciences Physiques, Grenoble (1983).

[14] BARRET, Ph., Régimes transitoires des machines tournantes électriques - Cours de l'ESE (Eyrolles Ed.) 1982. 